\title{
Collaborating for Competency-A Model for Single Electronic Health Record Onboarding for Medical Students Rotating among Separate Health Systems
}

\author{
Anne G. Pereira ${ }^{1}$ Michael Kim ${ }^{1} \quad$ Marcus Seywerd $^{2}$ \\ Minnesota Epic101 Collaborative*
}

Brooke Nesbitt ${ }^{3}$ Michael B. Pitt ${ }^{4}$ on behalf of the
Address for correspondence Anne G. Pereira, MD, MPH, Office of Medical Education, University of Minnesota Medical School, 420 Delaware Street SE, MMC 293, Minneapolis, MN 55455, United States (e-mail: apereira@umn.edu).

\section{Abstract}

Keywords

- clinical informatics

- professional training

- education and physician

- general health care providers

- training and education requirements

- education and strategies for health IT training

- education and certification
Background Use of the electronic health record (EHR) is widespread in academic medical centers, and hands-on EHR experience in medical school is essential for new residents to be able to meaningfully contribute to patient care. As system-specific EHR training is not portable across institutions-even when the same EHR platform is usedstudents rotating across health systems are often required to spend time away from clinical training to complete each system's, often duplicative, EHR training regardless of their competency within the EHR.

Methods We aimed to create a single competency-based Epic onboarding process that would be portable across all the institutions in which our medical students complete clinical rotations. In collaboration with six health systems, we created online EHR training modules using a systematic approach to curriculum development and created an assessment within the Epic practice environment.

Results All six collaborating health systems accepted successful completion of the developed assessment in lieu of standard site-specific medical student EHR training. In the pilot year, 443 students (94\%) completed the modules and assessment prior to their clinical training and successfully entered clinical rotations without time consuming, often repetitive onsite training, decreasing the cumulative time as student might be expected to engage in Epic onboarding as much as 20-fold.

Conclusion Medical schools with multisystem training sites with a single type of EHR can adopt this approach to minimize training burden for their learners and to allow them more time in the clinical setting with optimized access to the EHR.

\footnotetext{
* Minnesota Epic 101 Collaborative: E. Dewyre, K. Dean, C. Marshall, R. Miner, G. Olive, P. Papanno, V. Valentino, P. Varhol.
}

received

September 14, 2017

accepted after revision January 20, 2018
Copyright () 2018 Schattauer

DOI https://doi.org/

10.1055/s-0038-1635096.

ISSN 1869-0327. 


\section{Background and Significance}

In the past 15 years, the use of the electronic health record (EHR) has been widely adopted by the U.S. medical system and is now the norm in academic centers. Accordingly, medical educators must equip trainees with skills necessary to navigate the EHR efficiently and effectively. ${ }^{1}$ While recommendations underscore the importance of medical students having meaningful access to the EHR, its use presents unique challenges in undergraduate medical education. ${ }^{2}$ Systems struggle to find ways to allow students to have access necessary for training while protecting patients from potential harm. Health systems are generally risk averse, and due to a desire to protect patients and concerns about payer restrictions policies are created that limit medical students' roles within the EHR.

Students' access to the EHR ranges from a strictly observational role to documenting a parallel word-processing software, to being able to write orders to be released by a senior provider, and to document with cosignature. ${ }^{3}$ Possibly in part as a result of limited student access, challenges cited by residency programs indicate that we have not found a way to adequately train medical students on the use of the EHR. ${ }^{4,5}$ The central importance of this training is highlighted in two of the Association of American Medical College's Core Entrustable Professional Activities (EPA) for Entering Residency: EPA 4, enter and discuss orders and prescriptions, and EPA 5, document a clinical encounter in a patient record. ${ }^{6}$

Traditionally, student access to an EHR-no matter how limited-requires site-specific onboarding through in-person classes or electronic modules presented via learning management systems. ${ }^{7}$ This onboarding rarely, if ever, provides certification that is portable across health systems using the same EHR, and students are often granted access that varies across the systems through which they rotate within medical school, with less than half being able to document in patient charts in all the hospitals in which they train. ${ }^{8}$ We found no examples of systems that allow for portability of onboarding certification across institutions nor any that allow for competency-based assessment for students to demonstrate that they have skills necessary to avoid duplicative onboarding. The need for site-specific credentialing for EHR access can be time consuming and often redundant, occupying students' time that could be better spent in clinical training. While site-specific differences in EHR implementation likely create a need for unique components to onboarding, we sought to centralize the majority of onboarding with a single curriculum that could be accepted within multiple systems.

The University of Minnesota is one of the largest medical schools in the country with over 900 medical students who rotate through clinical experiences across several medical systems in the state. Most of these systems use Epic, and each site requires its own onboarding. We wished to determine the expectations and potential cumulative time burden of Epic onboarding for each of the sites where our students rotate. We then aimed to determine the feasibility of collaborating to form a single certification and competency-based assessment for medical students accepted by all teaching health systems in the state that use Epic.

\section{Methods}

We used Kern's Six Step Approach to Curriculum Development $^{9}$ as a framework to create our curriculum and assessment, entitled "Epic101."

Step 1-Identify problem/needs assessment. We surveyed the lead Epic educators responsible for training at the six health systems that use Epic and host medical students to understand required onboarding content, delivery method, and the expected time burden for learners. We also asked their willingness to join a collaborative effort to determine essential components for a single certification course. Based on the results of this survey, two authors (M.P. and M.S.) from the medical school and its affiliated health system planned to create modules and a competency-based assessment for a common certification course, vet them with the Epic educators, and oversee their implementation.

Step 2-Determine goals and objectives for the curriculum. We solicited topics that each group deemed important to include in this orientation and then refined this list with multiple iterations of a modified Delphi process via teleconferences and surveys. We used a 4-point scale (1-not necessary, 2-probably not needed, 3-probably needed, 4-essential) to assign relative importance to topics on our resulting list. Topics averaging a 3 or greater were included in the curriculum and are depicted in -Table 1.

Step 3-Identify an educational strategy. We chose online modules for several reasons: (1) this system was frequently used as part or all of the Epic onboarding across the systems, making buy-in and ability to collaboratively

Table 1 Agreed upon essential skills for Epic101 curriculum

\begin{tabular}{|l|l|}
\hline Skills & $\begin{array}{l}\text { Mean score of } \\
\text { importance for } \\
\text { inclusion (1-4 scale) }\end{array}$ \\
\hline Writing notes from scratch & 4.0 \\
\hline Using chart review & 4.0 \\
\hline Navigating results review & 3.9 \\
\hline Writing notes using SmartPhrases & 3.9 \\
\hline Finding your patient & 3.9 \\
\hline Reviewing the problem list & 3.6 \\
\hline Entering orders & 3.1 \\
\hline Using order sets & 3.1 \\
\hline $\begin{array}{l}\text { Performing medication } \\
\text { reconciliation }\end{array}$ & 3.1 \\
\hline Customizing a patient list & 3.1 \\
\hline Writing notes in NoteWriter & 3.0 \\
\hline $\begin{array}{l}\text { Using admission/discharge } \\
\text { navigator }\end{array}$ & 3.0 \\
\hline
\end{tabular}


develop the curriculum more feasible; (2) online modules could be housed behind a fire-wall requiring student logins which complies with Epic policies on use of proprietary screenshots from the EHR; (3) the online modules required a minimum of ongoing faculty and staff effort; (4) students were able to complete the curriculum asynchronously, thereby not interfering with their clinical experiences; and (5) online modules allow for an environment mimicking the EHR where learners can click and navigate as they would in the patient setting. We created four online modules that addressed the agreed upon content areas: Epic environment overview, order entry, patient information review, and chart documentation. We used the Adobe Captivate software to create the modules because the lead developer had experience with this platform. Lead educators from the six institutions vetted and refined the modules over a period of 4 months. Representative screenshots from these modules and full learning objectives are provided in -supplementary Material A (available in the online version).

Step 4-Develop an assessment. We aimed to create an assessment that would allow for demonstration of competency within Epic and that would ultimately grant portable certification across the participating institutions. We used the EpicPlay environment, a parallel, non-live EHR that simulates the Epic system and allows for the creation of simulated patients. This approach allowed us to create casebased questions mirroring real world encounters and to embed answers within simulated patient charts. Unlike module-based EHR assessments that require a certain pattern of clicking to demonstrate skill attainment, our approach allowed for multiple paths to each answer, mirroring the multiple real world paths to find such information within the EHR. We defined the ability to correctly answer $80 \%$ of twenty questions as demonstrating competency within Epic. An example of a series of questions is provided in the - Fig. 1. We provide the full assessment and key as - Supplementary Material B (available in the online version).

Step 5-Implementation. We placed the modules and assessment in the students' online learning management system in April 2016. In May 2016, all rising third year medical students preparing for their foundational clinical clerkships were required to complete both the modules and the assessment. All rising fourth year students who had already used Epic at least once had the option to take the assessment only, although they also had access to the modules.

Step 6-Evaluation and feedback. Upon completion of the modules, students completed an anonymous survey that

Patient Number 1 (Leah Beancurve; DOB 12/27/76)

You have just admitted a 40-year-old female with a diagnosis of pneumonia. You are preparing to present her to the team on rounds and you want to confirm a few things in her chart before your presentation.

Q1: You recall charting Leah's family history, but can't remember which conditions her father has. You turn to Epic to help you remember and see that her father has:
A. High cholesterol
B. Cancer
C. Diabetes
D. B and C
E. All of the above

Q2. You recall she has diabetes, but want to confirm which type by looking at her medical history. You confirm she has:
A. Diabetes insipidus
B. Type I diabetes mellitus
C. Type II diabetes mellitus
D. Gestational diabetes

Q3. While you are charting, a nurse pages you to ask the patient's code status. You check and find out that she:
A. Has a Do Not Resuscitate request
B. Is a Full Code

Fig. 1 Sample assessment questions requiring students to navigate simulated patients' chart. 
captured feedback on prior Epic experience, time requirement for module completion, level of comfort in using Epic upon entry into their first clinical rotation, and suggested future module topics. We also obtained feedback from all site Epic educators through regular email and phone communication. As a course-related activity with data collected as part of the routine class activity for program evaluation, the University of Minnesota Institutional Review Board considers the project exempt from review.

\section{Results}

Prior to implementation of the Epic101 curriculum, all six institutions had required their own EHR training regardless of any previous training a student might have completed. Four of the six had an online component to the training, and three had an in-person requirement with trainers at the elbow of the students. The time requirement ranged from less than 2 hours (one site) to more than 4 hours (two sites), with the majority requiring between 2 and 4 hours. With the training required at each site, sometimes for each rotation, an individual student could be required to spend up to 25 hours in duplicative onboarding, depending on his or her rotation schedule.

We obtained buy-in from all six institutions that if we collaborated to create a curriculum and assessment that addressed the overlapping expectations for each institution, each would allow this single onboarding to serve as the credentials for their respective site, eliminating or decreasing the amount of onsite training required. Representatives from each of the sites reviewed and provided feedback on content of each module. This was repeated until we had agreement that the module content adequately covered the skills listed in -Table 1. Examples of topics which did not reach the threshold for inclusion included InBasket Management and use of CareEverywhere to find patients' Epic records outside of the system. The workgroup discussed their inclusion and agreed they were not needed as core concepts for the medical student onboarding. Leadership then reviewed the drafted modules to ensure that it met their expectations before going live with the curriculum.

In the pilot year of the curriculum, 443 students (94\%) successfully completed the Epic101 assessment. All but three students did so on their first attempt, and of these, 438 students (99\%) completed the modules and assessment in less than 2 hours. A total of 173 students completed the postassessment survey (response rate 39\%), and of those, 125 (72\%) felt comfortable in their ability to use Epic effectively on their next clerkship.

As a result of the pilot year, five of the six sites have eliminated site-specific Epic training for students. One site still requires a half-hour onsite session for site-specific skills for inpatient clinical rotations. This change resulted in as much as a 20-fold reduction in time spent in Epic onboarding for students during their clerkships, allowing for more time in the clinical setting. After one year's experience, all six sites plan to use the curriculum and honor the completion certificate moving forward.

\section{Conclusion}

In our creation and pilot of the Epic101 curriculum, we demonstrated that multiple different clinical partners could overcome the challenges of institutional bureaucracies to create a portable curriculum that has been widely adopted, has saved time for both Epic trainers at clinical sites and students alike, and has provided students comfort in their readiness to use the EHR during their clinical rotations. Furthermore, our curriculum focuses on specific medical student tasks identified by the training institutions, in distinction to a broader array of skills addressed in a self-paced user guide an EHR software may incorporate within its platform.

This first cycle demonstrated the curriculum's feasibility with a $99 \%$ completion rate. It demonstrates a novel use of a preclinical competency-based assessment completed in the EHR environment in which the students will work. While module-based EHR assessments typically require students to follow an exact clicking algorithm to answer the question correctly, our assessment more closely resembles clinical practice in that it acknowledges that multiple roads lead to the same result within the EHR. This approach to assessment allows students the opportunity to demonstrate that they know how to find a laboratory result, for example, without being prescriptive in how they find it. While others have created vendor-agnostic student EHRs for training, ${ }^{10}$ the ability to leverage the EpicPlay environment, part of the out-of-the-box Epic platform, allows for a logical testing environment which mimics their eventual workspace. Additionally, we were able to create questions that went beyond simply data retrieval, or skills that could be accomplished by simply using the chart search feature. For example, we were able to assess the student's ability to successfully enter an order by asking details about what is shown when the order is pulled up (e.g., the Laboratory Code). If the student is able to successfully find this detail, it means he or she was able to find the order successfully.

We encountered several stakeholders along the way who felt it was unlikely we would be able to get six distinct systems to agree to a single onboarding process. Accordingly, our most significant outcome of this pilot project may be the fact that such a collaboration was, in fact, possible in the first place. We feel there are a few key factors that led to buy-in across institutions. First, we identified a common problem in the duplicative onboarding and offered an opportunity to collaborate for a solution. Second, we proposed that we (as the University site) would take the lead on creating the infrastructure of the modules, assessment, and tracking. Offering to take the burden of the logistics of making the curriculum, while keeping the equal stakes in input, may have contributed to the successful buy in. Additionally, we agreed to move forward with an "all for one, one for all" approach where we agreed that we would repeat feedback cycles until we were all in agreement that the modules met our needs. This so-called 
"Muska-Peer Pressure" helped move the project along as all the stakeholders were given equal content and veto power, yielding a true collaborative effort.

There are limitations in our work. First, the project was not designed as a controlled study, so while the majority of students (72\%) indicated they felt prepared to use Epic on day 1 , we are unable to compare their confidence with that of students who entered clinical rotations without this training. Second, survey questions were self-reported, so the comfort level may not reflect the skill level in EHR navigation. Third, as it is meant to replace the redundant onboarding addressing the logistics of Epic training, this curriculum focuses merely on the nuts and bolts of Epic use, not on the nuances of EHR such as professionalism, ability to maintain rapport with patients, etc. ${ }^{1,3}$ We do, however, address these important topics elsewhere in our medical school curriculum including opportunities to be evaluated on EHR use in simulated patient encounters as part of a more comprehensive curriculum addressing the provider's role in the EHR ecosphere. Fourth, the EpicPlay environment does not exactly mimic real Epic charts that have more content and granularity. However, it does provide an environment that is sufficiently challenging for novice users. Finally, we were not able to address certain aspects of training such as validating the correctness of individual student login and set-up for each health system. This is still a task that needs to be addressed by training teams at each health system.

This collaborative model serves as a proof of concept and is easily replicable by medical schools at which students rotate across multiple health systems. While use of this model necessitates a single type of EHR across multiple training sites, the consolidation of EHR vendors makes this situation likely around the country, and it would be possible to repeat this process for different EHR vendor cohorts within a training system. Our goal is that other programs with multisystem training sites with a single type of EHR will consider this approach to minimize training burden for their learners, thus allowing them more time in the clinical setting. The assessment relying on answers embedded in the charts of simulated patients within the EpicPlay environment requires minimal work to create and can likely be mirrored in other training programs. All six sites wish to continue this universal EHR certification and assessment, and we aim to continue this program for our residents as well as for our students, and to systematically gather feedback about comfort/EHR skill level during clinical experiences.

\section{Multiple Choice Questions}

1. Which of the following is a valuable conceptual framework to employ when developing a curricular intervention?
a. Community Centered Framework
b. Kern's Six Step Framework
c. Module Development Classification
d. Bloom's Escalation Inventory

Correct Answer: The correct answer is option b. Having a conceptual framework to serve as scaffolding for a curricular intervention is a valuable way to systematically approach the process of program development. The authors used Kern's Six Step Framework for Curriculum Development which lays out a process of performing a needs assessment, determining goals and objectives, choosing an educational strategy, developing an assessment, a plan for implementation, and evaluating the curriculum. Informaticists are often tasked with engaging learners around new innovation or processes, and being systematic in the development of this educational content is essential.

2. Which of the following did the authors cite as a benefit of embedding the assessment in the EHR Play Environment?

a. Allows for automatic grading of the assessment

b. Records the steps the learner took to answer a question

c. Allows for multiple avenues to arrive at a correct answer

d. Does not require Internet to access

Correct Answer: The correct answer is option c. Leveraging EpicPlay for the competency-based assessment allowed the authors to write question stems for simulated patients, with the answers embedded in their EHR records. This process, which differs from most module assessments that require a predefined click pattern to advance, allows for multiple avenues for the learner to navigate to arrive at the correct answer. For example, a question about a patient's laboratory results may be found via Chart Review, Results Review, 72Hour Laboratory flowsheet, etc. This process mimics the real world, whereby a user may take multiple different routes to find information in an EHR, and accordingly, is a true competency-based assessment rather than a test of memorizing where to click. This approach could be widely adopted by those tasked with creating onboarding curricula for EHR use.

Conflict of Interest

None.

\section{References}

1 Tierney MJ, Pageler NM, Kahana M, Pantaleoni JL, Longhurst CA. Medical education in the electronic medical record (EMR) era: benefits, challenges, and future directions. Acad Med 2013;88 (06):748-752

2 Hammoud MM, Dalymple JL, Christner JG, et al. Medical student documentation in electronic health records: a collaborative statement from the Alliance for Clinical Education. Teach Learn Med 2012;24(03):257-266

3 Hammoud MM, Margo K, Christner JG, Fisher J, Fischer SH, Pangaro LN. Opportunities and challenges in integrating electronic health records into undergraduate medical education: a national survey of clerkship directors. Teach Learn Med 2012; 24(03):219-224

4 Gagliardi JP, Turner DA. The electronic health record and education: Rethinking optimization. J Grad Med Educ 2016;8(03): 325-327 
5 Atwater AR, Rudd M, Brown A, et al. Developing teaching strategies in the EHR era: a survey of GME experts using the Delphi technique. J Grad Med Educ 2016;8(04):581-586

6 Association of American Medical Colleges. Core Entrustable Professional Activities for Entering Residency. Washington, DC: Association of American Medical Colleges updated 2014. Available at: https:// www.aamc.org/cepaer. Accessed July 31, 2017

7 Matson C, Stephens M, Steiner B, Kozakowski SM. Electronic health records: how will students learn if they can't practice? Ann Fam Med 2014;12(06):582-583
8 Friedman E, Sainte M, Fallar R. Taking note of the perceived value and impact of medical student chart documentation on education and patient care. Acad Med 2010;85(09):14401444

9 Thomas PA, Kern DE, Hughes MT, Chen BY, Eds. Curriculum Development for Medical Education. Baltimore: Johns Hopkins University Press; 2009

10 Elliott K, Judd T, McColl G. A student-centred electronic health record system for clinical education. Stud Health Technol Inform 2011;168:57-64 